\title{
An Iterative Finite Element Perturbation Method for Computing Electrostatic Field Distortions
}

\author{
Mohamed Boutaayamou, Ruth V. Sabariego and Patrick Dular
}

\begin{abstract}
A finite element (FE) perturbation method for computing electrostatic field distortions and electrostatic forces due to moving conductors is developed. First, an unperturbed problem (in the absence of conductors) is solved with the conventional FE method in the complete domain. Then, a perturbed problem is computed in a reduced region using the solution of the unperturbed problem as a source field. When the source is close to the perturbing regions, an iterative procedure may be required. The developed method allows for solving subproblems in reduced domains with independent problem-adapted meshes.
\end{abstract}

Index Terms-Electrostatic field distortions, finite element method, perturbation method.

\section{INTRODUCTION}

$\mathrm{T}$ he finite element (FE) modeling of an electrostatically driven moving conductor needs successive computations for each new position. This may be computationally expensive specially when dealing with 3D models of complex geometries. It is worth then benefiting from previous computations instead of starting a new complete FE solution for every new position.

The perturbation method has been shown to be of interest for computing field distortions and induced effects [1][2]. An unperturbed problem is first solved in a large mesh taking advantage of any symmetry and excluding additional regions and thus avoiding their mesh. Its solution is applied as a source to the further computations of the perturbed problems when conductive regions are added. It benefits from the use of different subproblem-adapted meshes, this way the computational efficiency increases as the size of each subproblem diminishes [2]. For some positions where the coupling between regions is significant, an iterative procedure is required to obtain an accurate solution. In [3], the perturbation method is applied to consider the introduction in a given domain of conductors subjected to a floating potential. A global-to-local method for static electric field calculations is presented in [4]. Herein, the mesh of the local domain is included in that of the global domain, whereas in the proposed perturbation method, the meshes of the perturbing regions are independent of the meshes of the unperturbed domain.

In this paper, the considered conductors are supposed to move with small speed in the absence of any magnetic field in

Manuscript received June 24, 2007. This work was supported by the Belgian French Community (ARC 03/08-298) and the Belgian Science Policy (IAP P6/21).

M. Boutaayamou, R. V. Sabariego and P. Dular are with the University of Liège, Dept. of Electrical Engineering and Computer Science, B28, B-4000 Liège, Belgium (e-mail: mboutaayamou@ulg.a.be). P. Dular is also with the Belgian National Fund for Scientific Research (F.N.R.S.). order to satisfy the static field assumption.

The perturbation method is developed here for solving electrostatic problems with an electric scalar potential FE formulation and computing the ensuing quantities, i.e. the electric field, electric charges and forces. This method is extended herein to account for the addition of conductors subjected to a fixed potential.

To illustrate the method, a 2-D FE computation of electrostatic field distortions between the plates of a capacitor is carried out.

\section{Strong Formulations}

\section{A. Unperturbed and Perturbed Electrostatic Problems}

We consider an electrostatic problem in a domain $\Omega$, with boundary $\partial \Omega=\Gamma_{e} \cup \Gamma_{d}$, of the 2-D or 3-D Euclidean space. The conductive parts of $\Omega$ are denoted $\Omega_{\mathrm{c}}$, with boundary $\partial \Omega_{c}$. Its governing differential equations and constitutive law in $\Omega$ are

$$
\operatorname{curl} \boldsymbol{e}=0, \operatorname{div} \boldsymbol{d}=q, \boldsymbol{d}=\varepsilon \boldsymbol{e},
$$

with associated boundary conditions (BCs)

$$
\boldsymbol{n} \times\left.\boldsymbol{e}\right|_{\Gamma_{e}}=0,\left.\boldsymbol{n} \cdot \boldsymbol{d}\right|_{\Gamma_{d}}=0,
$$

where $\boldsymbol{e}$ is the electric field, $\boldsymbol{d}$ is the electric flux density, $q$ is the electric charge density, $\varepsilon$ is the electric permittivity and $\boldsymbol{n}$ is the unit normal exterior to $\Omega$. According to (1a), the electric field $\boldsymbol{e}$ can be derived from an electric scalar potential $v$, i.e. $\boldsymbol{e}=-\operatorname{grad} v$.

Hereafter, the subscripts $u$ and $p$ refer to the unperturbed and perturbed quantities and associated domains, respectively. A so-called unperturbed electrostatic problem is first defined in $\Omega$ without considering the properties of a conductive region $\Omega_{c, p}$. Using the solution of the unperturbed problem as a source, the perturbed problem is solved in a domain $\Omega_{p}$, with boundary $\partial \Omega_{p}=\Gamma_{e_{p}} \cup \Gamma_{d_{p}}$, including the so-called perturbing conductive region $\Omega_{c, p}$ and its neighborhood. Indeed, electrostatic field distortions appear when the perturbing region $\Omega_{c, p}$ is added to the initial configuration.

At the discrete level, two independent meshes are used. A mesh of the whole domain in the absence of some additional conductive regions, and a mesh of the perturbing region. The electrostatic problem defined in each domain asks for mesh refinement of different regions [3].

\section{B. Perturbation Problem}

Particularizing (1a-b-c) and (2a-b) for both the unperturbed and perturbed quantities, and subtracting the unperturbed equations from the perturbed ones, a perturbation problem is defined in $\Omega_{p}$ (initially in $\Omega$ ) [1]-[3]. The obtained equations are expressed in terms of the field distortions $\boldsymbol{e}=\boldsymbol{e}_{p}-\boldsymbol{e}_{u}$ and $\boldsymbol{d}=\boldsymbol{d}_{p}-\boldsymbol{d}_{u}$ as the following 


$$
\begin{gathered}
\operatorname{curl} \boldsymbol{e}=0, \operatorname{div} \boldsymbol{d}=0, \boldsymbol{d}=\varepsilon_{p} \boldsymbol{e}+\left(\varepsilon_{p}-\varepsilon_{u}\right) \boldsymbol{e}_{u}, \quad(3 \mathrm{a}-\mathrm{b}-\mathrm{c}) \\
\boldsymbol{n} \times\left.\boldsymbol{e}\right|_{\Gamma_{e_{p}}}=0,\left.\boldsymbol{n} \cdot \boldsymbol{d}\right|_{\Gamma_{d_{p}}}=0 .
\end{gathered}
$$

In (3b), we assume that no charge density exists in $\Omega_{p}$. Note that in regions where $\varepsilon_{p} \neq \varepsilon_{u}$, an additional source term given by the unperturbed solution $\left(\varepsilon_{p}-\varepsilon_{u}\right) \boldsymbol{e}_{u}$ is considered in (3c). For the sake of simplicity, $\varepsilon_{p}$ and $\varepsilon_{u}$ are kept equal.

\section{Non-homogeneous BCs}

The homogeneous BCs to be considered in $\Omega_{p}$ are given in (4a-b). In case of non-counterbalanced electric charges, (4b) becomes non-homogeneous. The perturbed problem, with unknown fields $\boldsymbol{e}_{p}$ and $\boldsymbol{d}_{p}$, would require non-homogeneous conditions i.e.

$$
\boldsymbol{n} \times\left.\boldsymbol{e}_{p}\right|_{\Gamma_{e_{p}}}=\boldsymbol{n} \times\left.\boldsymbol{e}_{u}\right|_{\Gamma_{e_{p}}},\left.\boldsymbol{n} \cdot \boldsymbol{d}_{p}\right|_{\Gamma_{d_{p}}}=\left.\boldsymbol{n} \cdot \boldsymbol{d}_{u}\right|_{\Gamma_{d_{p}}} \cdot
$$

The unperturbed fields would thus serve as surface sources to be projected on the perturbed mesh boundary $\partial \Omega_{p}=\Gamma_{\mathrm{e}_{p}} \cup \Gamma_{d_{p}}$. However, such conditions can only be applied if a finite boundary $\partial \Omega_{p}$ is defined. Indeed, an infinite boundary would support a zero source[2].

For the perturbation problem, the following nonhomogeneous BCs have to be satisfied on $\partial \Omega_{p}$

$$
\boldsymbol{n} \times\left.\boldsymbol{e}\right|_{\Gamma_{e_{p}}}=-\boldsymbol{n} \times \boldsymbol{e}_{\boldsymbol{u}}||_{\Gamma_{p}},\left.\boldsymbol{n} \cdot \boldsymbol{d}\right|_{\Gamma_{d_{p}}}=-\left.\boldsymbol{n} \cdot \boldsymbol{d}_{\boldsymbol{u}}\right|_{\Gamma_{d_{p}}} \cdot(6 \mathrm{a}-\mathrm{b})
$$

According to (6a), the solution of the unperturbed problem $v_{u}$ can be used as a source for the perturbation problem. It reads

$$
v_{s}=v_{u} \mid \partial \Omega_{c, p} .
$$

The Neumann BC to be satisfied on $\Gamma_{d_{p}}$ is given by (6b). The projection of $v_{u}$ in a reduced support around some boundaries will be shown to be of interest for naturally evaluating $\boldsymbol{n} \cdot \boldsymbol{d}_{u}$, as well as for the calculation of the charges and forces.

\section{Weak Formulations}

\section{A. Unperturbed electric scalar potential as a source}

The unperturbed field distribution is first calculated in $\Omega$ as the solution of an electric scalar potential formulation, obtained from the weak form of the Laplace equation, i.e. $\operatorname{div}\left(-\varepsilon \operatorname{grad} v_{u}\right)=0$, as

$$
\begin{array}{r}
\left(-\varepsilon \operatorname{grad} v_{u}, \operatorname{grad} v^{\prime}\right)_{\Omega}-<\boldsymbol{n} \cdot \boldsymbol{d}_{u}, v^{\prime}>\Gamma_{d}=0, \\
\forall v^{\prime} \in \mathrm{F}(\Omega),
\end{array}
$$

where $(\cdot, \cdot)_{\Omega}$ and $\langle\cdot, \cdot\rangle_{\Gamma_{d}}$ denote a volume integral in $\Omega$ and a surface integral on $\Gamma_{d}$ of the product of its arguments; $F(\Omega)$ is the function space defined on $\Omega$ containing the basis functions for $v$ as well as for the test function $v^{\prime}$ [5]. At the discrete level, $\mathrm{F}(\Omega)$ is approximated with nodal FEs. The surface integral term in (8) can be associated with a global quantity or used for fixing a natural BC (usually homogeneous for a tangent electric field constraint) on a portion $\Gamma_{d}$ of the boundary of $\Omega$.

\section{B. Perturbation electric scalar potential problem}

The source of the perturbation problem $v_{s}$ is determined in the new added perturbing conductive region $\Omega_{c, p}$ through a projection method [6]. Given the conductive nature of the perturbing region, the projection of $v_{u}$ from its original mesh to that of $\Omega_{c, p}$ is limited to $\partial \Omega_{c, p}$. It reads

$<\operatorname{grad} v_{s}, \operatorname{grad} v^{\prime}>\partial \Omega_{c, p}-<\operatorname{grad} v_{u}, \operatorname{grad} v^{\prime}>\partial \Omega_{c, p}=0$,

$$
\forall v^{\prime} \in \mathrm{F}\left(\partial \Omega_{c, p}\right),
$$

where the function space $\mathrm{F}\left(\partial \Omega_{c, p}\right)$ contains $v_{s}$ and its associated test function $v^{\prime}$. At the discrete level, $v_{s}$ is discretized with nodal FEs and is associated to a gauge condition by fixing a nodal value in $\partial \Omega_{c, p}$.

When the unperturbed electric field value $\left(\boldsymbol{e}_{u}=-\operatorname{grad} v_{u}\right)$ is needed in the layer of FEs touching $\partial \Omega_{c, p}$ in $\Omega_{p} \backslash \Omega_{c, p}$, the projection of $v_{u}$ can be extended to this transition layer. This way, the computational effort of the projection is reduced. Indeed, having access to the value of $\boldsymbol{e}_{\boldsymbol{u}}$ in this layer allows to compute there the perturbed electric field $\boldsymbol{e}_{p}$, and thus both charges and electric forces can be calculated on $\partial \Omega_{c, p}$. Further, the projection should also be extended to the whole domain $\Omega_{c, p}$ in case of a dielectric perturbing region. We choose to directly project grad $v_{u}$ in order to assure a better numerical behaviour in the ensuing equations where the involved quantities are also gradients.

Substituting $v_{u}$ with $v$ in (8), the weak form of the perturbation electrostatic formulation defined in $\Omega_{p}$ reads

$$
\begin{array}{r}
\left(-\varepsilon \operatorname{grad} v, \operatorname{grad} v^{\prime}\right) \Omega_{\Omega_{p}}-<\boldsymbol{n} \cdot \boldsymbol{d}, v^{\prime}>\Gamma_{d_{p}}=0, \\
\forall v^{\prime} \in \mathrm{F}\left(\Omega_{p}\right),
\end{array}
$$

with Dirichlet BC $v=-v_{s} \mid \partial \Omega_{c, p}$.

\section{Iterative sequence of perturbation problems}

For close relative positions where the coupling between the source and perturbing regions is significant, an accurate solution can be obtained via an iterative procedure that calculates successive perturbations not only from the initial source region to the added conductor but also from the latter to the former. Each region gives a suitable correction as a perturbation with an accuracy dependent of the fineness of its mesh.

For each iteration $i(i=0,1, \ldots)$, we determine the electric scalar potential $v_{2 i}$ in $\Omega$, with $v_{0}=v_{u}$. The projection of this solution from its original mesh to that of the added conductor $\Omega_{c, p}$ gives a source $v_{s, 2 i+1}$ for a perturbation problem. This way, we obtain a potential $v_{2 i+1}$ and an electric charge in $\partial \Omega_{c, p}$ that will counterbalance the potential and the electric charge in $\partial \Omega_{c}$. A new source $v_{s, 2 i+2}$ for the initial configuration has to be then calculated. This is done by projecting $v_{2 i+1}$ from its support mesh to that of $\Omega$. This projection can be limited to the layer of FEs touching $\partial \Omega_{c}$ in $\Omega \backslash \Omega_{c}$, denoted $\Omega_{\text {layer }}$, i.e.

$$
\begin{gathered}
\left(\operatorname{grad} v_{s, 2 i+2}, \operatorname{grad} v^{\prime}\right) \Omega_{\text {layer }}-\left(\operatorname{grad} v_{2 i+1}, \operatorname{grad} v^{\prime}\right) \Omega_{\text {layer }}=0, \\
\forall v^{\prime} \in \mathrm{F}\left(\Omega_{\text {layer }}\right) .
\end{gathered}
$$

A perturbation electric scalar potential problem is defined in $\Omega$ as

$$
\begin{array}{r}
\left(-\varepsilon \operatorname{grad} v_{2 i+2}, \operatorname{grad} v^{\prime}\right)_{\Omega}-<\boldsymbol{n} \cdot \boldsymbol{d}_{2 i+2}, v^{\prime}>_{\Gamma_{d}}=0, \\
\forall v^{\prime} \in \mathrm{F}(\Omega),
\end{array}
$$

with non-homogeneous Dirichlet BC $v_{2 i+2}=-\left.v_{s, 2 i+2}\right|_{\partial \Omega_{c}}$ and Neumann BC $\left.\boldsymbol{n} \cdot \boldsymbol{d}_{2 i+2}||_{\Gamma_{d}=-\boldsymbol{n} \cdot \boldsymbol{d}_{2 i+1}}\right|_{\Gamma_{d}}$. The latter is not known in a strong sense. The associated surface integral term in (12) can be evaluated rather via the weak formulation of problem $2 i+1$ now applied to $\Omega_{\text {layer }}$, as

$<\boldsymbol{n} \cdot \boldsymbol{d}_{2 i+2}, v^{\prime}>_{\Gamma_{d}}=<-\boldsymbol{n} \cdot \boldsymbol{d}_{2 i+1}, v^{\prime}>_{\Gamma_{d}}$

$$
\begin{aligned}
& =-\left(-\varepsilon \operatorname{grad} v_{s, 2 i+2}, \operatorname{grad} v^{\prime}\right) \Omega_{\text {layer }} \\
& \forall v^{\prime} \in \mathrm{F}\left(\Omega_{\text {layer }}\right) .
\end{aligned}
$$

This iterative process is repeated until convergence for a given tolerance. 


\section{Electrostatic charges}

A suitable treatment of the surface integral term in (10) consists in naturally defining a global electric charge in the weak sense [5]. Choosing a test function $v^{\prime}$ equal to one on $\partial \Omega_{c, p}$, the electric charge can be computed at the postprocessing stage and is given in average by the volume integral in (10) in the layer of FEs touching $\partial \Omega_{c, p}$ in $\Omega_{p} \backslash \Omega_{c, p}$ as

$$
\begin{array}{ll} 
& Q_{p}=Q_{u}+Q, \\
\text { with } & Q_{u}=-\left(-\varepsilon \operatorname{grad} v_{u}, \operatorname{grad} v^{\prime}\right) \Omega_{p}, \\
\text { and } & Q=-\left(-\varepsilon \operatorname{grad} v, \operatorname{grad} v^{\prime}\right) \Omega_{p} .
\end{array}
$$

In case of iterative sequence of perturbation problems, the total charge on $\partial \Omega_{c, p}$ is given by the summation of elementary charges obtained in each iteration i.

\section{E. Electrostatic forces}

As previously mentioned, the perturbed electric field $\boldsymbol{e}_{p}$ can be computed in the layer of FEs touching $\partial \Omega_{c, p}$ in $\Omega_{p} \backslash \Omega_{c, p}$, The electric force distribution is calculated thus by locally applying the virtual work principle [7] in this transition layer. At the discrete level, the force at each node is obtained by deriving the electric energy in the considered layer of FEs with respect to a virtual displacement. The contribution of a reference element $\Delta$ to the force in a given direction is

$$
F_{r}=\int_{\Delta}\left[-\varepsilon \boldsymbol{e}_{p} J^{-1} \frac{\partial J}{\partial u} \boldsymbol{e}_{p}+\boldsymbol{e}_{p} \boldsymbol{e}_{p} \frac{\partial|J|}{\partial u}\right] \mathrm{d} \Delta,
$$

for a virtual displacement $r$ in this direction. $J$ is the geometrical Jacobian matrix with determinant $|J|$.

Given the non-linearity of the force, a direct summation of the forces at each iteration is not possible. The total electric field $\boldsymbol{e}_{p}$ has to be computed first at each iteration. The total force is then calculated by (15).

\section{Application}

In order to illustrate and validate the iterative perturbation method for electrostatic field distortions, we consider a parallel-plate capacitor (plate length and plate separation $200 \mu \mathrm{m})$. The conductive parts $\Omega_{c}$ of the capacitor are two electrodes between which the difference of electric potential is $\Delta \mathrm{V}=1 \mathrm{~V}$ (upper electrode fixed to $1 \mathrm{~V}$ ). A square conductor (side $=20 \mu \mathrm{m}$ ), fixed at $0.7 \mathrm{~V}$, is considered as perturbing region $\Omega_{c, p}$ inside the capacitor. The geometry of this conductor accounts for fringing field effects. Its distance from electrode at $\mathrm{V}_{0}=1 \mathrm{~V}$ is denoted $d$.

Fig. 1 shows example of meshes for the unperturbed and perturbation problems. An adapted mesh, specially fine in the vicinity of the corners of the perturbing conductor is used. Note that any intersection of perturbation problem boundaries with the unperturbed problem material regions is allowed.

Fig. 2 illustrates the sequence of associated solutions to be considered with the developed perturbation method, i.e. the unperturbed potential $v_{u}$, the perturbation potential $v$ and electric field $\boldsymbol{e}$, and the perturbed potential $v_{p}$ and electric field $\boldsymbol{e}_{\boldsymbol{p}}$.

For the position $d=20 \mu \mathrm{m}$, a sequence of perturbation problems has been carried out. The relative error of $v_{p}$ and the $y$-component of $\boldsymbol{e}_{p}$ computed near the conductor in some iterations is depicted in Fig. 3.
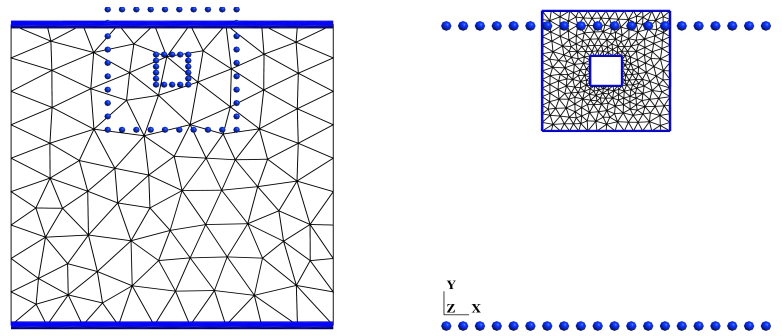

Fig. 1. Mesh of $\Omega$ (left) and adapted mesh of $\Omega_{p}($ right $)$.

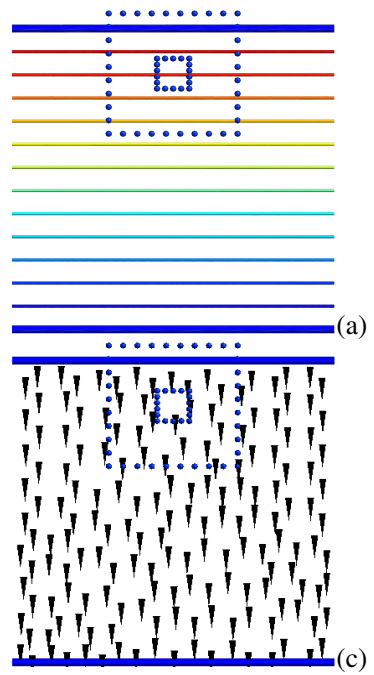

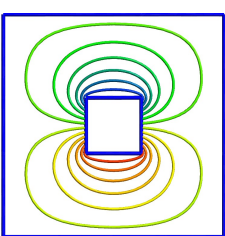

$\left(b_{1}\right)$

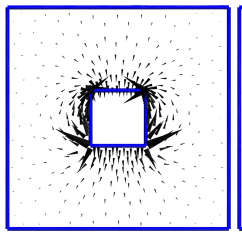

$\left(d_{1}\right)$

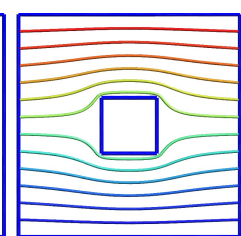

$\left(b_{2}\right)$



$\left(\mathrm{d}_{2}\right)$
Fig. 2. The unperturbed electric potential $v_{u}$ (a) and electric field $\boldsymbol{e}_{u}$ (c); the perturbation potential $v\left(\mathrm{~b}_{1}\right)$ and electric field $\boldsymbol{e}\left(\mathrm{d}_{1}\right)$; the perturbed potential $v_{p}$ $\left(\mathrm{b}_{2}\right)$ and electric field $\boldsymbol{e}_{p}\left(\mathrm{~d}_{2}\right)$.

The relative error for $v_{p}$ and $\boldsymbol{e}_{p}$ is $1 \%$ at iteration 15 and 23 , respectively. Note that $v_{p}$ converge faster than $\boldsymbol{e}_{p}$ (Fig. 3). Applying the Aitken acceleration to the iterative procedure speeds up the convergence. In that case, only 5 iterations are required for both $v_{p}$ and $\boldsymbol{e}_{p}$ (Fig. 4).
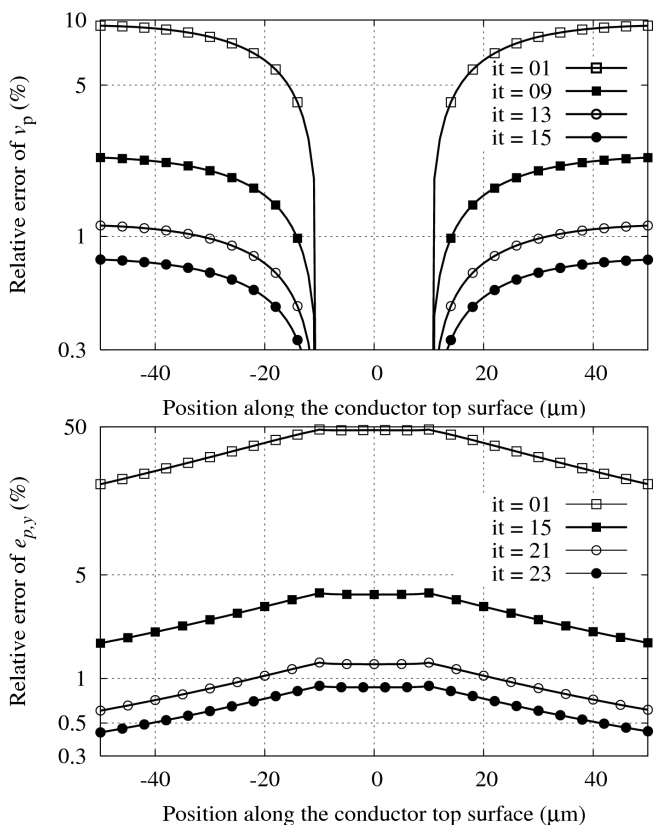

Fig. 3. Relative error of $v_{p}$ (top) and $e_{p}$ (y-component) (bottom) computed along the conductor top surface for some iterations. 




Position along the conductor top surface $(\mu \mathrm{m})$

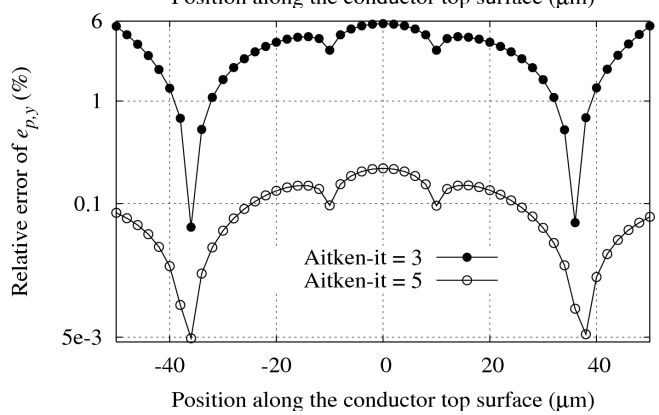

Fig. 4. Relative error of $v_{p}$ (top) and $e_{p}$ (y-component) (bottom) computed along the conductor top surface for some iterations with Aitken acceleration.

At odd iterations, the electric charge $Q_{p}$ and the electric forces on the outer surface of the conductor are computed. In order to compare with the FE solution, we consider the summation $F_{p}$ of the values of the y-component (dominant component) of the electric forces at each node of half the sides of the conductor from one corner facing electrode at $1 \mathrm{~V}$. Fig. 5 shows the convergence of $Q_{p}$ and $F_{p}$ as a function of iteration i.

An accuracy of $1 \%$ for $Q_{p}$ and $F_{p}$ is achieved at iteration 25 and 27, respectively. Note that $Q_{p}$ and $F_{p}$ have converged nearly at the same time as $\boldsymbol{e}_{p}$. When the Aitken acceleration is used, the number of iterations is reduced to 5 and 7 , respectively.

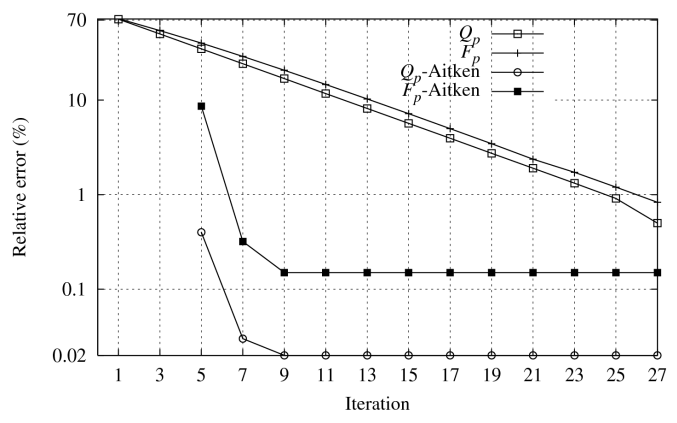

Fig. 5. Relative error of $Q_{p}$ and $F_{p}$ as a function of iteration number i.

Several relative positions $d$ of the conductor are listed in Table. I. For each of them, the perturbation problem is solved and an iterative process is carried out till convergence (i.e. the relative error of $v_{p}, e_{p}, Q_{p}$ and $F_{p}$ is smaller than $1 \%$ ). The number of iterations to achieve convergence without and with Aitken acceleration is shown. The latter is given between parentheses.

As expected, more iterations are needed when the conductor is closer to an electrode. It can be seen that $e_{p}, Q_{p}$ and $F_{p}$ need nearly the same number of iterations to achieve convergence at each considered position $d$. Furthermore, Aitken acceleration is very efficient.

TABLE. I

ITERATION NUMBERS TO ACHIEVE THE CONVERGENCE AS A FUNCTION OF THE DISTANCE BETWEEN THE CONDUCTOR AT $0.7 \mathrm{~V}$ AND ELECTRODE AT $1 \mathrm{~V}$.

\begin{tabular}{crrrl}
\hline$d(\mu \mathrm{m})$ & $v_{p}(\mathrm{~V})$ & $\boldsymbol{e}_{p}(\mathrm{~V} / \mathrm{m})$ & $F_{p}(\mathrm{~N})$ & $Q_{p}(\mathrm{C})$ \\
\hline 5 & $39(7)$ & $61(7)$ & $69(9)$ & $61(9)$ \\
10 & $25(5)$ & $37(7)$ & $43(9)$ & $39(9)$ \\
20 & $15(3)$ & $23(5)$ & $27(7)$ & $25(7)$ \\
50 & $1(-)$ & $5(5)$ & $7(7)$ & $19(7)$ \\
\hline
\end{tabular}

\section{Conclusion}

An iterative finite element perturbation method has been developed for efficiently computing electrostatic field distortions and forces due to moving conductors. First, an unperturbed problem (in the absence of some perturbing regions) is solved with the conventional FE method in the complete domain. Second, a perturbation problem is solved in a reduced region with an additional conductor using the solution of the unperturbed problem as a source. This approach uses independent meshes which are adapted for each associated problem. A projection from one mesh to another is used to feed a given problem with its sources. Benefits of the projections in reduced supports around some boundaries are pointed out. This way, charges and forces are computed.

For close relative positions where the coupling between the source and perturbing regions is significant, an accurate solution can be obtained via an iterative procedure.

As test case, a capacitor with a perturbing conductor subjected to a fixed potential has been studied. The application of Aitken acceleration to the iterative process has been proven to be very efficient.

\section{REFERENCES}

[1] Z. Badics et al., "An effective 3-D finite element scheme for computing electromagnetic field distortions due to defects in eddy-current nondestructive evaluation", IEEE Trans. Magn., vol. 33, no. 2, pp. 10121020, 1997.

[2] P. Dular and R.V. Sabariego, "A perturbation finite element method for modeling moving conductive and magnetic regions without remeshing", COMPEL, vol. 26, no. 3, pp. 700-711, 2007.

[3] M. Boutaayamou, R.V. Sabariego, P. Dular, "A Perturbation Method for the 3D Finite Element Modeling of Electrostatically Driven MEMS", $8^{\text {th }}$ Proc. EuroSimE 2007, London, pp. 50-54, 2007.

[4] I. Sebestyen, "Electric Field Calculation for HV Insulators Using Domain Decomposition Method", IEEE Trans. Magn., vol. 38, no. 2, pp. 1213-1216, 2002.

[5] P. Dular, W. Legros, A. Nicolet, "Coupling of local and global quantities in various finite element formulations and its application to electrostatics, magnetostatics and magnetodynamics", IEEE Trans. Magn., vol. 34, no. 5, pp. 3078-3081, 1998.

[6] C. Geuzaine, B. Meys, F. Henrotte, P. Dular, W. Legros, "A Galerkin projection method for mixed finite elements", IEEE Trans. Magn., vol. 35, no. 3, pp. 1438-1441, 1999.

[7] J. L. Coulomb and G. Meunier, "Finite element implementation of virtual work principle for magnetic or electric force and torque computation”, IEEE Trans. Magn., vol. 20, no. 5 , pp. 1894-1896, 1984. 